\title{
Assessing Collaborative Modeling Quality Based on Modeling Artifacts
}

\author{
D. (Denis) Ssebuggwawo ${ }^{1}$, S.J.B.A. (Stijn) Hoppenbrouwers ${ }^{1}$, \\ and H.A. (Erik) Proper $^{1,2}$ \\ 1 Institute of Computing and Information Sciences, Radboud University Nijmegen \\ Heyendaalseweg 135, 6525 AJ Nijmegen, The Netherlands, EU \\ D.Ssebuggwawo@science.ru.nl, stijnh@cs.ru.nl \\ 2 Public Research Centre - Henri Tudor, Luxembourg, EU \\ erik.proper@tudor.lu
}

\begin{abstract}
Collaborative modeling uses and produces modeling artifacts whose quality can help us gauge the effectiveness and efficiency of the modeling process. Such artifacts include the modeling language, the modeling procedure, the products and the support tool or medium. To effectively assess the quality of any collaborative modeling process, the (inter-) dependencies of these artifacts and their effect on modeling process quality need to be analyzed. Although a number of research studies have assessed and measured the quality of collaborative processes, no formal (causal) model has been developed to assess the quality of the collaborative modeling process through a combination of modeling artifacts. This paper develops a Collaborative Modeling Process Quality (CMPQ) construct for assessing the quality of collaborative modeling. A modeling session involving 107 students was used to validate and measure the quality constructs in the model.
\end{abstract}

Keywords: Collaborative Modeling, Modeling Process Quality, Modeling Artifacts, Instrument Validation, Structural Equation Modeling.

\section{Introduction}

Collaborative modeling, including the modeling of enterprises [123] and/or of associated business processes [4], brings together stakeholders with varying degrees of knowledge, expertise, skills and competencies. Such collaborative modeling, which is conceptually similar to group model building [5], brings with it a number of benefits and advantages. Although such benefits and advantages have been recognized in the literature, e.g. considerable productivity and improved results 6, substantiating the success of these collaborative efforts is far from trivial.

A number of factors come into play and need to be analyzed if we are to effectively and efficiently measure and evaluate modeling process quality and determine the success of a collaborative effort [7]. First, the different stakeholders have different priorities and preferences which need to be reconciled in a group

P. van Bommel et al. (Eds.): PoEM 2010, LNBIP 68, pp. 76-90, 2010.

(C) IFIP International Federation for Information Processing 20I0 
problem-solving activity, especially during evaluation of the modeling process. Second, a number of modeling artifacts are used in, and produced or amended during, the modeling process. These include the modeling language, the methods or approaches used to solve the problem, the intermediary and end-products produced and the medium or support tool that may be used to aid the collaboration. All these do impact on the success of the collaborative modeling effort and on the quality of the modeling process (especially its effectiveness and efficiency). Although the quality of each of these may be established separately, the quality of the entire modeling process is an aggregation of the quality of all these modeling artifacts.

While a number of approaches have been developed to measure and evaluate the quality of a collaborative modeling process, e.g. its successfulness [8] and users' satisfaction 9, there has not been any study that integrates the assessment of various modeling artifacts to determine the quality of a collaborative modeling process. Driven by the need to determine the efficiency and effectiveness of the modeling process, we propose an evaluation method that indeed integrates the assessments as an alternative method for determining the quality and successfulness of, and users' satisfaction with, a modeling process. To this effect, we develop and test a Collaborative Modeling Process Quality Assessment (CMPQ) construct - a causal model for assessing the quality of the modeling artifacts. We also present a validated instrument that can be used to measure the constructs in the model.

\section{Collaborative Modeling Artifacts and Their Quality Constructs}

Our approach is anchored on the Technology Acceptance Model (TAM) of Davis [10] and tries to assess the modelers' affective attitudes and perceptions of the quality of the modeling language, the end-products (models), the ease-of-use of a support tool and the usefulness of the modeling procedure. We therefore, identify and define the quality constructs selected to measure these perceptions for each of the modeling artifacts. It is not possible to include all the quality dimensions available in the literature for the modeling artifacts in our discussion. We, however, believe that those selected are a good representation of the modeling artifact quality. Many of the quality dimensions, of these constructs are discussed in [11. The operationalization of the quality dimensions identified in this section will be provided in Sect. 4 and the evaluation approach will be along the lines of the Method Evaluation Model described by Moody et al. [12].

\subsection{Modeling Language}

Many conceptual models, which are abstract representation of real world domains, are a collection of linked graphic symbols of an underlying modeling language. To Evaluate the model for quality, one needs to first look at the adequacy 
(expressive power, completeness, correctness, etc.) of the modeling language. Additionally, the evaluation of the adequacy of the modeling language should also take the perspective of the participant. She should be able to understand the concepts in the language, the concepts should be easy to learn and remember, the language should have a set of signs and symbols for producing the model and it should have well-defined rules for combining signs and symbols. We follow the generic quality framework in [13] to assess the quality of the modeling language. In view of this, we define the modeling language quality construct as follows.

The Perceived Quality of the Modeling Language (PQML) is the user's affective attitude towards a modeling language in providing a syntactic or domain meta language that provides concepts in which modelers define the problem, express and communicate the solution.

Quality dimensions for the PQML construct are defined in Table 1.

Table 1. PQML Construct Quality Dimensions

\begin{tabular}{l|l}
\hline Quality Dimension & Definition \\
\hline Understandability & $\begin{array}{l}\text { Understandability refers to how adequate the model represents concepts you recognize in view of your or } \\
\text { someone else's domain knowledge. } \\
\text { Clarity }\end{array}$ \\
$\begin{array}{l}\text { Clarity of the modeling language refers to how easily you learn and remember the concepts and notations of } \\
\text { the modeling language through the signs, symbols, textual expressions of the modeling language. } \\
\text { The syntax is the common agreed communication language for agents in a collaborative modeling process } \\
\text { and establishes a set of signs which can be exchanged and rules (syntactical rules) governing how the signs } \\
\text { can be combined. The syntax is related to the formal relations of signs to one another. }\end{array}$ \\
$\begin{array}{l}\text { Conceptual } \\
\text { minimalism }\end{array}$ \\
$\begin{array}{l}\text { Conceptual minimalism refers to the existence of primitive (basic) signs and symbols for representing data } \\
\text { concepts of the domain as separate objects and assembling the objects to form composite abstractions. }\end{array}$ \\
\hline
\end{tabular}

\subsection{Modeling Procedure}

Any performed task is driven by set goals [14. A goal is a result that a stakeholder strives to achieve and its awareness is accompanied by a set of perceived goal attainments [15. This means that Stakeholders in any collaborative modeling effort strive to achieve set goals. To achieve these goals, there must be a well-defined procedure in which they formulate and define the problem and agree on how the solution will be reached. To evaluate and measure the quality of the modeling procedure, one needs to assess whether the group goal is achieved. The most prominent measure for this is effectiveness. In [16], this is viewed as "...the extent to which a result contributes to the establishment of a goal set for the collaboration process" ( p.3).

Other quality constructs include e.g. the amount of time to reach the solution and to attain the goals and objectives, time to negotiate, etc. Stakeholders should also be satisfied with the negotiation, the decision and decision making process [17, the communication process and the goals and objectives set and how they are achieved through the modeling procedure. Stakeholders' commitment to supporting the goals and objectives, the collective decisions and their 
contribution to shared understanding is another measure of the success of a collaborative effort. In view of this discussion, we define the modeling procedure quality construct and its quality dimensions as follows.

Perceived Usefulness of the Modeling Procedure (PUMP) is the user's affective attitude towards the usefulness of the procedure used to detail the processes of how the problem is defined and how the solution is reached.

Quality dimensions for the PQML construct are defined in Table 2 .

Table 2. PUMP Construct Quality Dimensions

\begin{tabular}{l|l}
\hline Quality Dimension & Definition \\
\hline Efficiency & $\begin{array}{l}\text { Efficiency of the modeling procedure refers to the resources, e.g., time, required for reaching the solution and } \\
\text { attaining the modeling goals and objectives; the time needed to negotiate, reach agreement and consensus. } \\
\text { Effectiveness }\end{array}$ \\
$\begin{array}{l}\text { Modeling procedure effectiveness refers to how the modeling procedure enables the modelers in using } \\
\text { communication and negotiation to get the expected outcome and thus attain their set goals. It also includes the } \\
\text { facilitation and the way the modeling process is carried out and/or conducted, and the decision-making } \\
\text { process. }\end{array}$ \\
$\begin{array}{l}\text { Satisfaction of the modeling procedure refers to the modelers' positive feeling about the achievement of the } \\
\text { intended result using the modeling procedure. Intended results may include intermediary or end-results. } \\
\text { Satisfaction can concern the way modelers communicated, negotiated, reached agreement and how they made } \\
\text { modeling decisions. }\end{array}$ \\
Shared Understanding \\
$\begin{array}{l}\text { Commitment and shared understanding refer to the modeler's stake and promise to support the goals and } \\
\text { objectives of the modeling process, the responsibility to abide by the modeling rules and group decisions and } \\
\text { his/her readiness to contribute to the group's shared understanding. }\end{array}$
\end{tabular}

\subsection{End Products}

The end products are the results or outcomes of a collaborative modeling process. Where the modeling language is used to generate the products, these products are the models formed. Quality constructs for measuring and assessing the quality of the modeling process outcome include product quality which may include the complexity, abstractness, clarity, correctness, completeness, consistency and understandability of the products, see, for example, [18]. In addition to product quality, modelers should be satisfied with both the process that generates the products and the outcome [19], they should be satisfied with the syntactic, semantic, pragmatic, empirical and physical quality of both the modeling language and the model. In case of models, they should be modifiable and maintainable, i.e. they should be easily changed and re-used. We use the general framework defined in 20] for the assessment of quality of models. The definition of the end-products quality construct in the context of this paper is given next.

The Perceived Quality of the End Products (PQEP) (models) is the user's affective attitude towards the outcome (including intermediary and final models) of a modeling process.

Quality dimensions for the PQML construct are defined in Table 3 . 
Table 3. PQEP Construct Quality Dimensions

\begin{tabular}{|c|c|}
\hline Quality Dimension & Definition \\
\hline Understandability & $\begin{array}{l}\text { Understandability of the products refers to the degree to which the modelers comprehend the language } \\
\text { concepts represented in the products, e.g., its syntax, semantics, etc., the relationship between the different } \\
\text { concepts which are depicted by the products, and the ease with which the modelers can explain the } \\
\text { concepts in the products even to those who never participated in the modeling process. }\end{array}$ \\
\hline $\begin{array}{l}\text { Modifiability and } \\
\text { Maintainability }\end{array}$ & $\begin{array}{l}\text { Modifiability and maintainability of the products refer to ease of changing the products to accommodate } \\
\text { new changes and the degree to which the products can be kept up-to-date, and how easily they can be re- } \\
\text { used in the re-engineering and re-structuring of the enterprise processes. }\end{array}$ \\
\hline Satisfaction & $\begin{array}{l}\text { Product satisfaction of the modelers refers to a positive feeling about the product's quality. This could } \\
\text { include satisfaction with respect to the product's correctness, completeness, accuracy, consistency, clarity, } \\
\text { understandability and/or its complexity. }\end{array}$ \\
\hline
\end{tabular}

\subsection{Support-Tool: The Medium}

The support tool or the medium is the means that supports and facilitates the collaborative modeling process. This can range from a simple white-board to a group support system (GSS) 21]. To evaluate such a support tool, a number of quality constructs are used. These include the enjoyment and/or fun derived from the use of the support tool 22]; functionality of the tool and its usability 23]. An excellent survey concerning the use of technology and its impact on the performance of groups is given in [24. The support tool is also required to facilitate the collaboration and communication process, e.g. the negotiation process and decision making process. We define the support-tool or medium construct for assessing a collaborative modeling process as follows.

The ease of use of the medium (EOUM) (or the support tool (EOUST)) is the user's affective attitude towards a technology-based group support system (GSS) that supports the collaborative modeling process.

Quality dimensions for the PQML construct are defined in Table 4.

Table 4. EOUM Construct Quality Dimensions

\begin{tabular}{|c|c|}
\hline Quality Dimension & Definition \\
\hline Functionality & $\begin{array}{l}\text { Tool functionality refers to the different functions that a tool has which support activities of the modeling } \\
\text { process. It also refers to how the support tool executes the modeling activities and how reliable it is in } \\
\text { executing those activities. }\end{array}$ \\
\hline Usability & $\begin{array}{l}\text { Usability of a tool support refers to its effectiveness and efficiency to achieve specified goals in particular } \\
\text { environments. It is a set of attributes which bear on the effort needed for use and on the individual assessment } \\
\text { of such use by a stated or implied set of users. Where efficiency relates to the level of effectiveness achieved } \\
\text { to the expenditure of resources whereas effectiveness refers to the goals or sub-goals of using the support tool } \\
\text { to the accuracy and completeness with which these goals can be achieved. }\end{array}$ \\
\hline $\begin{array}{l}\text { Satisfaction \& } \\
\text { Enjoyment }\end{array}$ & $\begin{array}{l}\text { Satisfaction refers to perceived usability of the support tool by its users and the acceptability of the support } \\
\text { tool to the people who use it and to other people affected by its use. It also refers to the degree of fun and } \\
\text { enjoyment by the modelers in using the tool. Measures of satisfaction may relate to specific aspects of the } \\
\text { system or may be measures of satisfaction with the overall support system. }\end{array}$ \\
\hline
\end{tabular}




\section{$3 \quad$ Modeling Experiment Set-Up}

This part of the paper discusses the following: description of the modeling experiment and subjects and the modeling task.

- Modeling Experiment and Subjects. To assess the quality of the CMPQ construct, we conducted a modeling session. The subjects were third-year undergraduate (day and evening) students offering a Bachelor of Information Technology and Computing (BITC) degree course. The modeling experiment was conducted after an introductory course in information and system modeling using UML. A simple UML editor, embedded within the Collaborative Modeling Architecture (COMA) tool 25], was used. A total of 107 students participated in the modeling experiment. They were divided into 6 groups with an average of 17 participants and 3 or 4 participants per computer terminal. The modeling experiments were conducted on two days, each day having three groups. Each experiment lasted for not more than 70 minutes.

- Task Description. The modeling case that was given to the students concerned procurement of medical drugs and equipment by the Pharmacy and Medical Equipment Department of a University Teaching Hospital and distributing these to the different wards and departments of the University Teaching Hospital. The students' task was to: 1) identify the different processes, associated activities and objects; 2) develop the conceptual model using COMA's UML editor; and 3) assess the quality of the whole collaborative modeling process by filling-out the given questionnaire immediately after the modeling session. Task 1 is associated with the modeling procedure whose quality is assessed via the PUMP construct. Task 2 is concerned with the end-products (conceptual model), the modeling language (UML) and the medium or support tool (COMA) whose quality is measured, respectively, via the PQEP, PQML and EOUM constructs. Task 3 is concerned with assessing the quality of the whole collaborative modeling process via the CMPQ construct by filling-out the given questionnaire. The development, reliability and validity testing of this CMPQ Construct Measurement Instrument is described next.

\section{CMPQ Construct Measurement Instrument}

Evaluating and measuring the quality of the collaborative modeling process through the modeling artifacts presented in Sec. 2 requires a validated instrument. We did a literature survey for previously validated instruments narrowing the scope to only those assessing: 1) collaborative modeling quality, 2) users' satisfaction and 3) success of a collaborative effort within collaborative modeling and/or group model building. This survey revealed that validated instruments presented in [15/16 17/26/27] satisfy at least one of the selection criteria given above and the first is the closest to this study. Unfortunately, being from the area of Collaboration Engineering, it looks at collaboration patterns. Many of the quality constructs presented in all these studies provide a background to the quality dimensions discussed in this paper. Although the assessment done 
therein, tries to get users' perception of quality, successfulness, etc, and satisfaction with product, process, system, decision and the decision making process, we propose an alternative way of performing the assessment.

Our assessment approach evaluates quality of the collaborative effort through the quality of the modeling artifacts: the modeling language, the modeling procedure, the end-products and the support tool or medium. Specifically, we look at the PQML, PUMP, PQEP and EOUM constructs to develop an integrated approach and a construct for assessing the quality of the collaborative modeling process. Although Straub 28] advises to use previously validated research instruments wherever possible, he warns that a major modification to a research instrument will negatively affect its validity and reliability. None of the validated instruments above could satisfy our needs and alteration was not possible since many of the questions on the instruments for similar constructs needed rephrasing to suit our needs. In light of the above observations, it was not possible to adopt or alter any of the existing research instruments. It is for this reason that we developed a new instrument.

\subsection{Content Validation of the Instrument}

We developed a new instrument to assess and measure Collaborative Modeling Process Quality(CMPQ) through four constructs: PQML, PUMP, PQEP and EOUM. The initial measurement instrument had a total of 44 quality dimensions synthesized from the literature: 10 for PQML, 10 for PUMP, 15 for PQEP and 9 for EOUM. These are shown in the second column of the table in Appendix A.

Content Validation. Content validity, which is established through literature reviews and/or expert panels or judges, measures the degree to which the selected items in the research instrument represent the content pool to which the research instrument will be generalized [29]. A panel of three content experts is considered adequate for content validation 30. In light of this recommendation, three experts were asked for their judgement about the adequacy and representation of the constructs and their quality dimensions for the CMPQ construct. A 5-point rating scale (with $1=$ Highly Appropriate and $5=$ Highly Inappropriate) was used to rate the appropriateness of the quality dimensions. The mean value of each of the dimensions ranged between 1.10 and 4.33. This means that some of the quality dimensions were inappropriate.

The qualitative judgement of the experts indicated that the numbers above were too many for any construct and many were found to overlap. It was recommended to refine, merge and group many of these quality constructs. The original dimensions and their refined and merged groupings are shown in Appendix A, The groupings form a set of sixteen quality dimensions for each of the four modeling constructs defined in Sec. 2. The instrument with sixteen questions measuring the quality of the constructs, using a 7-point scale (with $1=$ strongly agree and $7=$ strongly disagree), is given in Appendix B 


\subsection{Exploratory Factor Analysis}

We carried out an Exploratory Factor Analysis (EFA) subjecting the 107 case in the data set to Principal Component Analysis (PCA) 31]. The Promax rotation method was used since the data exhibited strong correlations among the extracted factors. To identify the suitable number of factors underlying the CMPQ construct we used the three recommended steps in 32. We dropped all factors with at most 0.4 values. This condition prevented cross-loading on more than one factor at 0.4 and above. We also applied and repeated factor analysis using $3,4,5$ and 6 factor loadings. All factors were extracted at eigenvalue of 1 . The 4-factor loadings was found to be the most suitable for the CMPQ construct and explains $70.3 \%$ of the variance. The factor loadings of the 16 quality dimensions of the four quality constructs measured through the research instrument are given in Table 5 ,

Table 5. Factor Analysis and Reliability Results

\begin{tabular}{|c|c|c|c|c|c|c|c|}
\hline & \multirow{2}{*}{\multicolumn{4}{|c|}{ Factor }} & \\
\hline & & & & & & & \multirow{2}{*}{$\begin{array}{c}\text { Cronbach's } \\
\alpha\end{array}$} \\
\hline Construct & Code & Quality Dimension & 1 & 2 & 3 & 4 & \\
\hline PQML & $\begin{array}{l}\text { ML1 } \\
\text { ML2 } \\
\text { ML3 } \\
\text { ML4 }\end{array}$ & $\begin{array}{l}\text { Understandability } \\
\text { Clarity } \\
\text { Syntax Correctness } \\
\text { Conceptual Minimalism }\end{array}$ & $\begin{array}{l}.895 \\
.798 \\
.886 \\
.787\end{array}$ & & & & .866 \\
\hline PUMP & $\begin{array}{l}\text { MP5 } \\
\text { MP6 } \\
\text { MP7 } \\
\text { MP8 }\end{array}$ & $\begin{array}{l}\text { Efficiency } \\
\text { Effectiveness } \\
\text { Satisfaction } \\
\text { Commitment \& Shared Understanding }\end{array}$ & & $\begin{array}{l}.718 \\
.883 \\
.842 \\
.882\end{array}$ & & & .850 \\
\hline PQEP & $\begin{array}{l}\text { EP9 } \\
\text { EP10 } \\
\text { EP11 } \\
\text { EP12 }\end{array}$ & $\begin{array}{l}\text { Product Quality } \\
\text { Understandability } \\
\text { Modifiability \& Maintainability } \\
\text { Satisfaction }\end{array}$ & & & $\begin{array}{l}.833 \\
.840 \\
.795 \\
.795\end{array}$ & & .834 \\
\hline EOUM & $\begin{array}{l}\text { ST13 } \\
\text { ST14 } \\
\text { ST15 } \\
\text { ST16 }\end{array}$ & $\begin{array}{l}\text { Functionality } \\
\text { Usability } \\
\text { Satisfaction \& Enjoyment } \\
\text { Collaboration Communication \& } \\
\text { Facilitation }\end{array}$ & & & & $\begin{array}{l}.817 \\
.944 \\
.702 \\
.661\end{array}$ & .833 \\
\hline & & \begin{tabular}{|l|} 
Eigenvalue \\
Cumulative Variance Explained (\%) \\
\end{tabular} & $\begin{array}{l}4.57 \\
28.6\end{array}$ & $\begin{array}{l}2.68 \\
45.3\end{array}$ & $\begin{array}{l}2.35 \\
60.0\end{array}$ & $\begin{array}{l}1.65 \\
70.3 \\
\end{array}$ & \\
\hline
\end{tabular}

Extraction Method: Principal Component Analysis. Rotation Method: Promax with Kaiser Normalization. Rotation converged in 5 iterations. 
Reliability Tests and Construct Validity. We note from the results presented that all factor loadings of the 16 items load on a single factor for each of the PQML, PUMP, PQEP and EOUM constructs. This is preliminary evidence of uni-dimensional reliability of the research instrument, in the Exploratory Factor Analysis (EFA) method using the Principal Component (PCA) technique, where item measures reflect only one underlying trait or concept 33. To further check the scientific rigor of the research instrument, and to confirm our a-priori assumptions about the reliability (internal consistency) of the research instrument, we computed the Cronbach's alpha values [34. The computed values, as is evident in Table [5] were all above the threshold value of 0.60 or 0.70 recommended for the EFA method using the the PCA technique 35. This is proof that the quality dimensions of the PQML, PUMP, PQEP and EOUM constructs are related to each other within the same construct and these variables are consistent in measuring each of these constructs.

To check whether the research instrument is an effective measure of the CMPQ theoretical construct, we had to check the instrument for Construct validity which is established through either discriminant, convergent or factorial validity [36. The presence of eigenvalues of or above 1, loadings of at least 0.40 and no cross-loadings above 0.40 , is confirmation of discriminant, convergent and factorial validity and hence confirms construct validity for EFA method using the PCA technique [29. As is evident from Table 5], the eigenvalues are above 1.0 and all loadings are above the threshold value and there are no cross-loadings for the 4-factor model used. Therefore the research instrument is an effective measure of the CMPQ construct.

\subsection{Confirmatory Factor Analysis}

In the previous section a data-driven and theory development method, EFA 37. was used to develop and identify the patterns of relationships between the PQML, PUMP, PQEP, and EOUM constructs and their quality dimensions in measuring the $\mathrm{CMPQ}$ construct. To further confirm the identified patterns of relationships between the constructs measuring the CMPQ construct, and test the theory of these relationships, we carried out a Confirmatory Factor Analysis on the data set 38. CFA being a special case of Structural Equation Modeling (SEM) 39] requires special tools. We used AMOS 18.0 40, on the data set used in EFA by applying the maximum likelihood (ML) method. We developed two models, a proposed conceptual and theoretical model (Model1) and a competing model (Model2), that included the four identified constructs: PQML, PUMP, PQEP and EOUM as first order factors in the first model and CMPQ as a second-order factor in the second model onto which the four factors in model 1 load. These models are presented in Fig. 1. The second model was to act as a competing model for the first model and is intended to corroborate the four factors in the first model [41. Results of the CFA analysis are shown in Table 6.

As can be seen CFA results confirm the construct validity and reliability of the result instrument, since the values of GFI, NFI, AGFI are close to the threshold values and Cronbach's alpha for AMOS is above 0.70 [3335. 

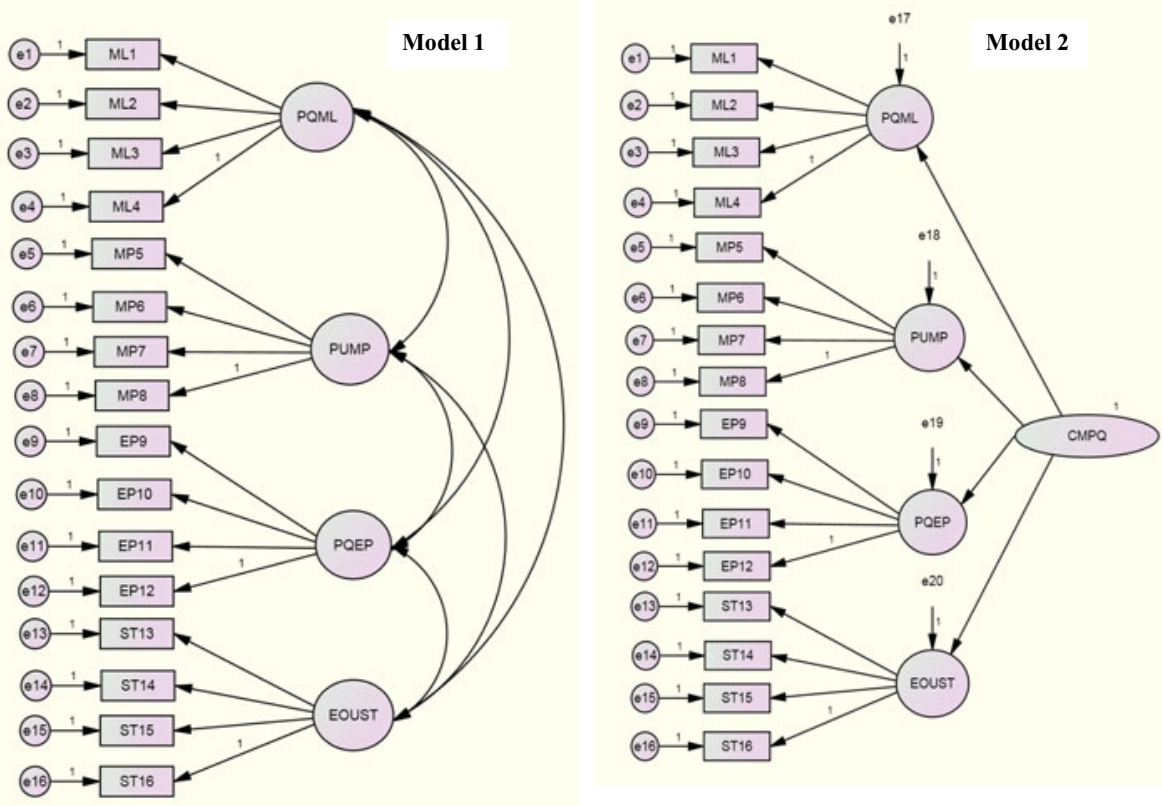

Fig. 1. CFA Models: Conceptual Model and Competing Model

Table 6. Factor Loadings and Model Fit Test Results

\begin{tabular}{|c|c|c|c|c|c|c|c|}
\hline \multirow[t]{2}{*}{ Construct } & \multirow[t]{2}{*}{ Code } & \multirow[t]{2}{*}{ Quality Dimension } & \multicolumn{2}{|c|}{ Factor Loading } & \multicolumn{3}{|c|}{ Model Fit Indices } \\
\hline & & & Model 1 & Model 2 & Fit index: Threshold & Model 1 & Model 2 \\
\hline \multirow{4}{*}{ PQML } & ML1 & Understandability & 0.76 & 0.77 & : SB & 142.923 & 143.738 \\
\hline & ML2 & Clarity & 0.75 & 0.75 & & 98 & 100 \\
\hline & ML3 & Syntax Correctness & 0.84 & 0.84 & $\mathrm{p}$-value $: \mathrm{p}<0.05$ & 0.002 & 0.003 \\
\hline & ML4 & Conceptual Minimalism & 0.80 & 0.80 & $\chi^{2} /$ d.f $: 1<\chi^{2} /$ d.f $<3$ & 1.458 & 1.437 \\
\hline \multirow{8}{*}{ PUMP } & & & & & RMR $\quad:<0.10$ & 0.129 & 0.131 \\
\hline & & & & & $:>0.90$ & 0.863 & 0.861 \\
\hline & MP5 & Efficiency & 0.62 & 0.62 & & & \\
\hline & MP6 & Effectiveness & 0.94 & 0.94 & AGFI $:>0.80$ & 0.810 & 0.813 \\
\hline & MP7 & Satisfaction & 0.71 & 0.71 & NFI $:>0.90$ & 0.837 & 0.815 \\
\hline & MP8 & Commitment \& Shared Understanding & 0.78 & 0.78 & TLI $\quad:>0.90$ & 0.931 & 0.912 \\
\hline & & & & & CFI $:>0.90$ & 0.942 & 0.923 \\
\hline & & & & & RMSEA : $<0.08$ & 0.066 & 0.064 \\
\hline \multirow{4}{*}{ PQEP } & EP9 & Product Quality & 0.78 & 0.76 & & & \\
\hline & EP10 & Understandability & 0.77 & 0.81 & : SB & 218.923 & 215.738 \\
\hline & EP11 & Modifiability \& Maintainability & 0.07 & 0.71 & CAIC : SB & 358.490 & 347.960 \\
\hline & EP12 & Satisfaction & 0.74 & 0.71 & \multirow{6}{*}{\multicolumn{3}{|c|}{$\begin{array}{l}\text { Key } \\
\text { SB : Smaller is Better. }\end{array}$}} \\
\hline \multirow{5}{*}{ EOUM } & & & & & & & \\
\hline & ST13 & Functionality & 0.68 & 0.68 & & & \\
\hline & ST14 & Usability & 0.67 & 0.67 & & & \\
\hline & ST15 & Satisfaction \& Enjoyment & 0.80 & 0.81 & & & \\
\hline & ST16 & Collaboration Communication \& Facilitation & 0.81 & 0.80 & & & \\
\hline
\end{tabular}




\section{Discussion}

The first observation about the results of CFA is that the (standardized) factor loadings of the the conceptual model (Model 1) and the competing model (Model 2) are close. In fact they are the same for the PQML and PUMP constructs while slight differences are noticed for the PQEP and EOUM. This closeness of the results indicates that the Model used in the EFA was a good conceptual model. To determine the possibility of Model 2 being preferred to Model 1, we compare the model fit indices of both models to determine which ones are near or better than the threshold values (see 29|33|36|41] for these threshold values).

Comparison of the fit indices indicates that the values are close. Model 1 has better fit indices than model 2 for the following indices: chi-square value $\left(\chi^{2}\right)$, degrees of freedom (d.f), probability value (p-value), chi-square to degree of freedom ratio $\left(\chi^{2} /\right.$ d.f $)$, root-mean square residual (RMR), goodness-of-fit index (GFI), Normed fit index (NFI), Tucker-Lewis index (TLI) and comparative fit index (CFI). Model 2 has better fit indices than Model 1 for the following indices: adjusted goodness-of-fit index (AGFI), root-mean square-error for approximation (RMSEA), Akaike information criterion (AIC) and consistent Akaike information criterion(CAIC). The fit index values of both models for RMR, GFI and NFI are below the threshold values. Since the AIC value of Model 2 is better than Model 1, Model 2 is the most parsimonious model 39] and this means is preferred to Model 1. However, looking at the number of fit indices above the threshold values for Model 1 which is higher than model 2, it is equally plausible to conclude that Model 1 is better. We believe reaching a convincing conclusion requires further analysis using different sets of data for the EFA and CFA. We, however, believe that Model 1 could be used if (inter-)dependencies between the quality constructs PQML, PUMP, PQEP and EOUM are of interest. Model 2 could be used if particular explanatory relationships (latent regressions) [39 are postulated among the quality constructs rather than analysing only the (inter)relationships among the quality constructs as is the case for model 1.

Theoretical and Practical Implications. One of the theoretical implications of this research is that a conceptual domain for the CMPQ construct has been defined based on the modeling artifacts used in and produced during the modeling process together with their quality dimensions. Rather than assessing the quality of the modeling process by defining quality dimensions directly for the CMPQ construct, these could be defined for the PQML, PUMP, PQEP and EOUM and the quality assessed via these constructs. This approach has been operationalized by applying the EFA and CFA methods which have, respectively, produced and confirmed the existence of measurable quality indicators for the four quality constructs. The practical implication of the study is that the developed research instrument offers a means of assessing and measuring the quality of the CMPQ construct. This can be used by collaborative modelers and facilitators to assess their perceived quality, usefulness or ease of use of not only the modeling process and the outcomes, but also the modeling language and the support tool or medium. 
Limitations of the Study. The limitation of the study is that we used the same data set for both EFA and CFA. To explicitly determine which of the models presented is better, we need to have two separate data sets. Due to the problem of getting the required minimum sample size this was not possible. Moreover, splitting the one data set into two data sets: one for EFA and the other for CFA was not possible since this would drastically reduce the sample size. Use of students to evaluate the modeling process using the questionnaire could not guarantee that the results were the best we had hoped for. Using professional modelers could be the best but these are hard to come by!

\section{Conclusion and Future Research}

This paper set out to develop a quality construct for assessing the quality of collaborative modeling through four constructs: PQML, PUMP, PQEP and EOUM. It has been shown through known statistical techniques: Exploratory Factor Analysis (EFA) and Confirmatory Factor Analysis (CFA) that the approach is sound and the research instrument passes the validity and reliability tests. The contribution of this paper is thus two-fold. First, it develops a method of assessing collaborative modeling quality based on modeling artifacts used in, and developed during the collaborative modeling effort. The approach developed assesses users' perceived quality, perceived usefulness and ease of use of the modeling artifacts. Second, a validated instrument for measuring the developed constructs and assessing the quality of the $\mathrm{CMPQ}$ construct is presented. We hope this research will stimulate further academic interest in the study of assessing the quality of the collaborative modeling process via the modeling artifacts that are used and produced during the modeling process. Our future research is to further develop and test the theory about the (inter-)dependencies and explanatory relationships between different constructs in relation to the CMPQ construct.

\section{References}

1. Barjis, J., Kolfschoten, G.L., Verbraeck, A.: Collaborative Enterprise Modeling. In: Proper, E., Harmsen, F.,, J.L. (eds.) PRET 2009, LNBIP, pp. 50-62. Springer, Heidelberg (2009)

2. Stirna, J., Persson, A.: Ten Years Plus with EKD: Reflections from Using an Enterprise Modeling Method in Practice. In: Proper, H.A., Halpin, T.A., Krogstie, J. (eds.) Proceedings of the EMMSAD 2007, held in conjunctiun with CAiSE 2007, pp. 97-106. Tapir Academic Press, Trondheim (2007)

3. Gjersvik, R., Krogstie, J., Følstad, A.: Participatory Development of Enterprise Process Models. In: Krogstie, J., Halpin, T., Siau, K. (eds.) Information Modeling Methods and Methodologies, pp. 195-215. IGI Global (2005)

4. Rittgen, P.: Collaborative Modeling of Business Processes-A Comparative Case Study. In: Proceedings of the 24th Annual ACM Symposium on Applied Computing, Waikiki Beach, Honolulu, Hawaii, pp. 225-230. ACM, New York (2009)

5. Vennix, J.A.M.: Group Model Building: Facilitating Team Learning Using System Dynamics. Wiley, Chichester (1996) 
6. Vreede, G.J., de Briggs, R.O.: Collaboration Engineering: Designing Repeatable Processes for High-Value Collaborative Task. In: Proceedings of the 38th HICSS Conference, IEEE computer Society Press, Los Alamitos (2005)

7. Sedera, W., Rosemann, M., Doebeli, G.: A Process Modelling Success Model: Insights From A Case Study. In: 11th ECIS Conference, Naples, Italy, pp. 1-11 (2003)

8. Davison, R.: An Instrument for Measuring Meeting Success: Revalidation and Modification. Information and Management 36, 321-328 (1999)

9. Briggs, R.O., Reinig, B.A., de Vreede, G.J.: Meeting Satisfaction for Technology Supported Groups: An Empirical Validation of a Goal-Attainment Model. Small Group Research 37 (2006)

10. Davis, F.D.: Perceived Usefulness, Perceived Ease of Use and User Acceptance of Information Technology. MIS Quarterly 13(3), 319-340 (1989)

11. Ssebuggwawo, D., Stijn Hoppenbrouwers, S.J.B.A., Proper, H.A.: Evaluating Modeling Sessions Using the Analytic Hierarchy Process. In: Persson, A., Stirna, J. (eds.) PoEM 2009, LNBIP, vol. 39, pp. 69-83. Springer, Heidelberg (2009)

12. Moody, D., Sindre, G., Brasethvik, T., Sølvberg, A.: Evaluating the Quality of Process Models: Empirical Analysis of a Quality Framework. In: Spaccapietra, S., March, S.T., Kambayashi, Y. (eds.) ER 2002. LNCS, vol. 2503, p. 380. Springer, Heidelberg (2002)

13. Nysetvold, A.G., Krogstie, J.: Assessing Business Process Modeling Languages Using a Generic Quality Framework. In: Halpin, T., Krogstie, J., Siau, K. (eds.) Proceedings of the EMMSAD 2005 held in conjuction with CAiSE 2005, Porto, Lisboa, pp. 545-556. Idea Group, USA (2005)

14. Locke, E.A., Latham, G.P.: A Theory of Goal Setting and Task Performance. Prentice Hall, Eaglewood Cliffs (1990)

15. Briggs, R.O., de Vreede, G.J., Reinig, B.A.: A Theory and Measurement of Meeting Satisfaction. In: Proccedings of the 26th HICCS Conference (HICCS 2003), p. 25c . IEEE Computer Society, Los Alamitos (2003)

16. Duivenvoorde, G.P.J., Kolfschoten, G.L., Briggs, R.O., de Vreede, G.J.: Towards an Instrument to Measure the Successfulness of Collaborative Effort from the Participant Perspective. In: Proceedings of the 42nd HICCS Conference (HICCS 2009), pp. 1-9. IEEE Computer Society, Los Alamitos (2009)

17. Paul, S., Seetharaman, P., Ramamurthy, K.: User Satisfaction with System, Decision Process and Outcome in GDSS-Based Meeting: An Experimental Investigation. In: Proceedings of the 37th HICSS Conference (HICSS 2004), pp. 37-46. IEEE Computer Society, Washington (2004)

18. Dean, D.L., Orwig, R.E., Vogel, D.R.: Facilitation Methods for Collaborative Modeling Tools. Group Decision and Negotiation 9, 109-127 (2000)

19. Reinig, B.A: Towards Understanding of Satisfaction with the Process and Outcomes of Teamwork. Journal of MIS 19, 65-83 (2003)

20. Krogstie, J.: A Semiotic Approach to Quality in Requirements Specifications. In: Proceedings of the IFIP TC8 /WG8.1 Working Conference on Organizational Semiotics: Evolving a Science of Information Systems, Montreal, Canada, pp. 231-249. Kluwer B.V., The Netherlands (2001)

21. Dean, D.L., Orwig, R.E., Vogel, D.R.: Technological Support for Group Process Modeling. Journal of MIS 11(3), 43-64 (1994)

22. de Vreede, G.J.: Collaborative Business Engineering with Animated Electronic Meetings. Journal of MIS 14(3), 141-164 (1997)

23. Fjermestad, J., Hiltz, S.R.: A Decsriptive Evaluation of Group Support Systems and Case Field Studies. Journal of MIS 17, 115-159 (2001) 
24. Fjermestad, J., Hiltz, S.R.: An Assessment of Group Support Systems. Experimental Research: Methodology and Results. Journal of MIS 15, 7-149 (1999)

25. Rittgen, P.: Collaborative Modelling Architecture (COMA), http://www.coma.nu/COMA_Tool.pdf (accessed on: 09/08/2010)

26. den Hengst, M., Dean, D.L., Kolfschoten, G.L., Chakrapani, A.: Assessing the Quality of Collaborative Processes. In: Proceedings of the 39th Annual HICCS Conference (HICCS 2006), vol. 1, p. 16b . IEEE Computer Society, Los Alamitos (2006)

27. Green, S.G., Taber, T.D.: The Effect of Three Social Decision Schemes on Decision Group Processes. Organizational Behaviour and Human Performance 25(1), 97-106 (1980)

28. Straub, D.W.: Validating Instruments in MIS Research. MIS Quarterly 13, 47-169 (1989)

29. Straub, D., Boudreau, M.-C., Gefen, D.: Validation Guidelines for IS Positivist Research. Communication of the AIS 13, 380-427 (2004)

30. Lynn, M.: Determination and Quantification of Content Validity. Nursing Research 35(6), 382-385 (1986)

31. Joliffe, I.T.: Principal Component Analysis, 2nd edn. Springer Series in Statistics. Springer, Heidelberg (2002)

32. Tabachnick, B.G., Fidell, L.S.: Using Multivariate Statistics, 4th edn. Harper Collins, New York (2001)

33. Gefen, D., Straub, D., Boudreau, M.-C.: Structural Equation Modeling and Regression Guidelines for Research Practice. Communication of the AIS 4(7), 1-78 (2000)

34. Cortina, J.M.: What is Coefficient Alpha? An Examination of Theory and Application. Journal of Applied Psychology 78, 98-104 (1993)

35. Nunnally, J.C.: Psychometric Theory 2nd edn, McGraw-Hill, New York (1978)

36. Hair, J.F., Tatham, R.L., Anderson, R.E., Black, W.: Multivariate Data Analysis, 5th edn. Prentice-Hall, Upper Saddle River (1998)

37. Brown, T.A.: Confirmatory Factor Analysis for Applied Research. Guilford Press, New York (2006)

38. Albright, J.J., Hun, M.P.: Confirmatory Factor Analysis Using Amos, LISREL, Mplus, and SAS/STAT CALIS. Technical Working Paper. The University Information Technology Services (UITS) Center for Statistical and Mathematical Computing, Indiana University, http://www.indiana.edu/all/cfa/cfa.pdf (accessed on: $17 / 08 / 2010)$

39. Raykov, T., Marcoulides, G.A.: A First Course in Structural Equation Modeling, 2nd edn. Psychology Group Taylor \& Francis Group, New York (2006)

40. Arbuckle, J.L.: AMOS 18: User's Guide. SPSS Inc., Chicago (2009)

41. Tojib, D.R., Sugiant, L., Sendjay, F.: User Satisfaction with Business-to-Employee Portals: Conceptualization and Scale Development. EJIS 17, 649-667 (2008) 


\section{A Original Quality Dimensions and Their Groupings}

\begin{tabular}{|l|l|l|}
\hline Construct & Original Quality Dimensions & Quality Dimension Groups \\
\hline PQML & $\begin{array}{l}\text { construct deficit, construct overload, construct redundancy, construct } \\
\text { excess; expressive power, directness, systematicity; syntax, semantic \& } \\
\text { pragmatic clarity; modeling primitive adequacy }\end{array}$ & $\begin{array}{l}\text { Understandability, Clarity, Syntax correctness, } \\
\text { Conceptual minimalism }\end{array}$ \\
\hline PUMP & $\begin{array}{l}\text { Efficiency; effectiveness; ease of application, in-out-description adequacy, } \\
\text { process \& relation description adequacy, method compatibility, interaction } \\
\text { \& collaboration adequacy, communication \& negotiation adequacy; rule \& } \\
\text { goal commitment, shared understanding }\end{array}$ & $\begin{array}{l}\text { Efficiency, Effectiveness, Satisfaction, } \\
\text { Commitment \& Shared Understanding }\end{array}$ \\
\hline PQEP & $\begin{array}{l}\text { correctness, completeness, propriety, clarity, consistency, orthogonality, } \\
\text { generality, syntax adherence adequacy, semantics adequacy, pragmatics } \\
\text { adequacy; user-comprehensibility; Modifiability, re-usability, flexibility; } \\
\text { user satisfaction }\end{array}$ & $\begin{array}{l}\text { Product Quality, Understandability, } \\
\text { Modifiability \& Maintainability, Satisfaction }\end{array}$ \\
\hline EOUM & $\begin{array}{l}\text { tool functionality, performance \& reliability; efficiency, effectiveness; } \\
\text { satisfaction; synchronicity, negotiation/argumentation adequacy, } \\
\text { commenting/proposition adequacy, planning/agenda setting adequacy }\end{array}$ & $\begin{array}{l}\text { Functionality, Usability, Satisfaction \& } \\
\text { Enjoyment, Collaboration \& Communication } \\
\text { Facilitation }\end{array}$ \\
\hline
\end{tabular}

\section{B Research Instrument}

\begin{tabular}{|c|c|c|c|c|}
\hline Code & Measurement Item & & \begin{tabular}{|l|l|l|l|l|l}
2 & 3 & 4 & 5 \\
\end{tabular} & \begin{tabular}{|l|l|l|l|l}
5 & 6 & 7
\end{tabular} \\
\hline \multicolumn{5}{|c|}{ Perceived Quality of Modeling Language (PQML) } \\
\hline ML1 & The modeling language was easy to understand. & & & \\
\hline ML2 & The modeling language was easy to learn and remember. & & & \\
\hline ML3 & There are expressions that are not allowed by the modeling language. & & & \\
\hline ML4 & It was easy to represent all concepts using the available signs and symbols of the modeling language & & & \\
\hline \multicolumn{5}{|c|}{ Perceived Usefulness of Modeling Procedure (PUMP) } \\
\hline MP5 & $\begin{array}{l}\text { We took a lot of time to negotiate, reach agreement and consensus and at times failed to make important } \\
\text { decisions. }\end{array}$ & & & \\
\hline MP6 & The modeling procedure enabled us to reach the solution and attain the modeling goal in less time. & & & \\
\hline MP7 & $\begin{array}{l}\text { I was satisfied with the way we communicated/negotiated, reached consensus and agreement and how we made } \\
\text { the modeling decisions to obtain the end results. }\end{array}$ & & & \\
\hline MP8 & $\begin{array}{l}\text { I was in full support of the goals and objectives, had a stake in achieving the goals and objectives of the of the } \\
\text { modeling session and contributed to shared understanding. }\end{array}$ & & & \\
\hline \multicolumn{5}{|c|}{ Perceived Quality of End-Product (PQEP) } \\
\hline EP9 & $\begin{array}{l}\text { The intermediary and end-products were accurate in depicting all the identified aspects of the domain and only } \\
\text { essential details were represented. }\end{array}$ & & & \\
\hline EP10 & $\begin{array}{l}\text { When I look at the final models I understand the rules and concepts represented and model is easy to understand } \\
\text { and explain to those that never participated in the modeling session. }\end{array}$ & & & \\
\hline EP11 & It is easy to modify the model to accommodate new changes, to re-use and restructure the model. & & & \\
\hline EP12 & I am satisfied with the quality of the intermediary and end-products. & & & \\
\hline \multicolumn{5}{|c|}{\begin{tabular}{|c|} 
Ease of Use of Medium (EOUM) \\
\end{tabular}} \\
\hline ST13 & $\begin{array}{l}\text { The modeling tool functionally supported and aided the modeling process and was reliable in executing the } \\
\text { modeling process . }\end{array}$ & & & \\
\hline ST14 & The modeling tool was efficient and effective in helping me realize the goals of the modeling process & & & \\
\hline ST15 & $\begin{array}{l}\text { I was satisfied with the functionality offered by the modeling tool, it was fun to use the modeling tool and I } \\
\text { enjoyed using it. }\end{array}$ & & & \\
\hline ST16 & $\begin{array}{l}\text { The modeling tool helped the group to collaboratively develop the model, reach agreement/consensus and our } \\
\text { communication was very much facilitated by the modeling tool. }\end{array}$ & & & \\
\hline
\end{tabular}

Scale: $1=$ Strongly agree, $4=$ Neutral, $7=$ Strongly disagre. 